\title{
Ototoxicity: A Challenge in Diagnosis and Treatment
}

\author{
Purushothaman Ganesan ${ }^{1}$, Jason Schmiedge ${ }^{1}$, Vinaya Manchaiah ${ }^{2,3,4}$, Simham Swapna $^{5}$, \\ Subhashini Dhandayutham ${ }^{6}$, and Purushothaman Pavanjur Kothandaraman ${ }^{7}$ \\ ${ }^{1}$ Expert Hearing Solutions, Wall Street Audiology Group Inc., Saskatoon, Canada \\ ${ }^{2}$ Department of Speech and Hearing Sciences, Lamar University, Beaumont, TX, USA \\ ${ }^{3}$ Department of Speech and Hearing, School of Allied Health Sciences, Manipal University, Manipal, \\ ${ }^{4}$ Audiology India, Mysore, \\ ${ }^{5}$ Hindu Mission Hospital, Chennai, \\ ${ }^{6}$ Madras ENT Research Foundation, Chennai, \\ ${ }^{7}$ Department of ENT, SRM Medical College Hospital and Research Centre, Chennai, India
}

\begin{abstract}
Received December 16, 2017
Revised January 10, 2018

Accepted January 16, 2018
\end{abstract}

Ototoxicity is the pharmacological adverse reaction affecting the inner ear or auditory nerve, characterized by cochlear or vestibular dysfunction. The panorama of drug-induced hearing loss has widened over last few decades. Although ototoxic medications play an imperative role in modern medicine, they have the capacity to cause harm and lead to significant morbidity. Evidence has shown early detection of toxicity through prospective ototoxicity monitoring allows for consideration of treatment modifications to minimize or prevent permanent hearing loss and balance impairment. Although many ototoxicity monitoring protocols exist, their practicality is questionable due to several factors. Even though the existing protocols have proven to be effective, certain lacunae in practice have been encountered due to discrepancies among recommended protocols. Implementation of these protocols is mostly held back due to the incapacitated status of the patient. The choice of early ototoxicity identification techniques is still debatable due to variables such as high degree of sensitivity, specificity and reliability, less time consumption and less labour-intensive to the patient. Hence, the diagnosis and effective treatment of ototoxicity is challenging, even today. A stringent protocol with more practicality encompassing all elements aimed at profiling the effects of ototoxicity is greatly needed. This review describes an efficient application of ototoxicity monitoring and treatment protocol as an attempt to reduce the challenges in diagnosis and management of ototoxicity.

J Audiol Otol 2018;22(2):59-68

KEY WORDS: Ototoxicity · High-frequency audiometry · Distortion product of otoacoustic emission · Ototoxicity monitoring.

\section{Introduction}

The panorama of potential ototoxicity of some antibiotics gained recognition among medical professionals after World War II [1]. With the evolution of the pharmaceutical industry over the decades, there has been an increase in the number of potential ototoxic agents in the international pharmacopeia. Ototoxicity is the cellular degeneration of cochlear and/or vestibular tissues leading to its functional deterioration, due

This is an Open Access article distributed under the terms of the Creative Commons Attribution Non-Commercial License (http://creativecommons.org/licenses/by-nc/4.0/) which permits unrestricted non-commercial use, distribution, and reproduction in any medium, provided the original work is properly cited. to the usage of certain therapeutic agents [2]. Ototoxic drugs can act on the cochlea, vestibular system or both. There are over 600 categories of drugs which have the potential to cause ototoxicity [3]. Aminoglycoside antibiotics, platinum-based chemotherapeutic agents, loop diuretics, macrolide antibiotics, and antimalarials are the commonly used ototoxic drugs [2] with well-documented efficacy against various infections and malignancies in children and adults.

Damage to the auditory system due to drugs can present in various ways: tinnitus, hearing loss, hyperacusis, aural fullness, dizziness, and vertigo [3]. Symptoms may range from temporary tinnitus to permanent deafness and/or mild imbalance to total incapacitation. The onset of these symptoms can be si- 
multaneous or singular. Symptoms can develop rapidly or gradually and can be either reversible or irreversible. The commencement of symptomatology is often uncertain. A high inter-individual variability in symptomatology is observed due to differences in genetic factors, pharmacokinetics, metabolic status of the individual and co-morbid medical conditions. Although the drug-induced hearing loss is not a life-threatening condition, it can have a negative impact on communication and health-related quality of life, with significant vocational, educational and social consequences [4]. In children, even minimal to mild hearing loss can hamper speech, language, cognitive and social development, which may lead to poor scholastic performance and psychosocial functioning. The goal of management of ototoxicity is to minimize or prevent communication impairment $[3,5]$, and plan appropriate rehabilitation measures.

\section{Epidemiology}

Ototoxicity occurs in all age groups. Although it is well documented, the global magnitude of its incidence is unknown due to various reasons such as existence of varied and diverse criteria to define ototoxicity [Chang grading system, Tune grading system, American Speech-Language-Hearing Association (ASHA) criteria etc.], wide range of reactions to a drug in different ethnic groups, utilization of various audiological protocols for evaluation, and lack of referral for otological symptoms since these are either reversible or non-life threatening. The cisplatin ototoxicity occurs between $23 \%$ and $50 \%$ in adults and up to $60 \%$ in children [4,6]. However, some studies have reported elevated hearing thresholds in up to $100 \%$ of cisplatin-treated cancer patients $[7,8]$, while it is estimated to be $63 \%$ with aminoglycosides and $6-7 \%$ with furosemide [9]. In addition, incidence severity of ototoxic hearing loss seems to be dose-dependent and cumulative in fashion and can be influenced by factors such as: age, gender, and co-morbid conditions like congestive heart failure, renal failure, hypertension, genetic susceptibility, geographic factors, type of drug, route of administration, duration of therapy, bio-availability and pre-existing hearing loss.

\section{Ototoxicity and Drug Metabolizing Genes}

Well defined unequivocal associations have been established between chemotherapy and its complications. However, significant inter-individual variability is observed in the development of therapy-related hearing loss for a given therapeutic exposure. These variations can likely be explained by genetic predisposition and its interaction with chemotherapy which can potentially exacerbate the toxic effect of treatment on normal tissues. In order to minimize at-risk individuals, it would be highly advantageous to identify genetic variants that predispose ototoxicity, but precise identification of these genetic variants presents a daunting task due to phenotypical variation.

Candidate gene pharmacogenetic studies have explored the relationship between drug-induced hearing loss and several genotypes such as thiopurine methyltransferase (TPMT), ATPbinding cassette transporter $\mathrm{C} 3(A B C C 3)$, glutathione-Stransferase subclasses (GSTP1, GSTM1, GSTT1), catechol-Omethyltransferase $(C O M T)$, and megalin, yielding largely inconsistent results [10-19]. To date, certain mutations in the mitochondrial DNA, the A1555G mutation, in particular, has been linked with increased susceptibility to aminoglycosiderelated ototoxicity [20]. A genome-wide association study approach has identified the association between cisplatin-induced hearing loss and genetic variants such as superoxide dismutase 2 (SOD2) and Acylphosphatase-2 (ACYP2) [21,22].

\section{Diagnosis}

A common problem encountered in clinical practice is a delay in diagnosing ototoxicity. Drug-induced hearing loss appears to be quite variable and highly inconsistent. Diagnosis should be based on the patient's history, symptoms and test results. Many variables such as age, co-morbid medical conditions, and cognition levels may delay the early detection of ototoxicity. It is essential to create awareness among patients, caretakers, and nurses about the significance of symptoms such as tinnitus, ear fullness, reduced hearing, oscillopsia, and disequilibrium, and that this should be immediately reported to their physicians and audiologists. Ototoxicity often progresses undetected until a considerable hearing communication problem becomes apparent signifying hearing deterioration in the frequencies necessary for speech understanding. Clinically, ototoxicity is diagnosed by comparing audiometric test results done before and after the administration of ototoxic drugs.

\section{Current practice of ototoxicity monitoring}

Ototoxic hearing loss can substantially impact interpersonal communication and quality of life, but its impact can be minimized by following an established ototoxicity monitoring program as it embraces the principles of early identification and early intervention. Ototoxicity monitoring is essential in obtaining a pathophysiological description of the ototoxic agent's effects and for keeping track of the changes over time [5]. Ototoxic hearing change has a relatively predictable course of action as it preferentially affects the basal turn of the co- 
chlea, its outer hair cells in particular (high-frequency limit of hearing) and progresses to the apical portion including lower speech frequencies. Ototoxic monitoring can be successful only when a fixed regimen is followed. This involves the education and coordinated effort of numerous health professionals (Oncologist, ENT specialist, audiologist, clinical pharmacist, nurses) and also patients. Monitoring techniques should be considered based on their efficacy, sensitivity, and specificity.

Sequential ototoxicity monitoring helps with the following:

1) Comparing the auditory test results during the course of drug therapy,

2) Early identification of change in hearing,

3) Need for potential alterations in therapy,

4) Prevention of debilitating ototoxic-induced hearing loss if therapy is changed, and

5) Auditory rehabilitation to minimize the negative impact of ototoxicity.

The ASHA's [23] "Guidelines for the audiologic management of individuals receiving cochleotoxic drug therapy (1994)" states that baseline audiometric test should be done within 24 hours of administering chemotherapeutic agents and within 72 hours of administering aminoglycoside antibiotics. Audiological reassessment done within 24 hours of the baseline test can determine patient reliability for behavioural threshold testing. It was also highlighted that testing should be initiated with a comprehensive case history including possible otologic disorders, co-morbid conditions, exposure to noise, family history of ear disorders/genetic susceptibility to ototoxic drugs, and prior usage of ototoxic medication. Ototoxicity typically begins in the frequencies above $8,000 \mathrm{~Hz}$ and progresses to lower speech frequencies [24]. Therefore, ASHA and the American Academy of Audiology (AAA) recommend that baseline assessment should include behavioural measures such as pure-tone audiometry (PTA) from $250 \mathrm{~Hz}$ to $8,000 \mathrm{~Hz}$ and high-frequency audiometry (HFA) from 9,000 $\mathrm{Hz}$ to $20,000 \mathrm{~Hz}$, plus objective measures such as distortion product of otoacoustic emissions (DPOAEs) and tympanometry, along with self-evaluating questionnaires [23,25]. Each measure provides valuable information in an ototoxicity monitoring program (peripheral and/or central auditory function, apical versus basal cochlear turn, and subjective versus objective measure), where test protocol selection is driven by both clinical purpose and patient epidemiological characteristics.

There is a wide variability between recommended audiometric tests for ototoxicity monitoring and tests actually utilized in clinical practice. There are a limited number of studies reporting the implementation of the protocol in ototoxicity monitoring in cancer patients. In the UK, Wilkinson and Mora
[26] performed postal questionnaire surveys of pediatric cystic fibrosis (CF) units to audit audiological surveillance in these services, and 22 out of 27 centres responded. Of the respondents, only about $41 \%$ of the centers had an established audiological screening protocol where baseline hearing assessments were carried out only in $2 / 22(9 \%)$ centers prior to ototoxic drug administration. A similar survey study done in the US concluded that routine audiometric evaluation was performed in only $22 / 84(26 \%)$ of the CF foundation accredited care centers and only 14 of those performed annual hearing evaluations [27]. Another UK-based study concluded that due to lack of clear protocols and guidelines, there was great difference in implementation of monitoring services (baseline testing, audiological tests used, criteria for referring at-risk patients, frequency and timing of monitoring, diagnostic criteria for ototoxicity and protocol for change in therapy) and significant uncertainty between all professionals involved [28]. A significant proportion of these professional groups agreed upon the fact that conventional PTA was the most frequently used tool for monitoring ototoxicity, followed by tympanometry. Unfortunately, HFA and DPOAEs, which are known to be more effective than PTA for early detection of ototoxicity [29,30], were sparsely used.

Despite the presence of substantial evidence supporting the significance of early identification of ototoxic-induced hearing loss, effective monitoring tools for ototoxicity have not been implemented in most clinical settings. Reasons for this are that these tools are expensive, the procedures are time-consuming and difficult to perform in a serial manner in chronically ill patients.

\section{Efficient application of ototoxicity monitoring protocol}

There is no doubt that ototoxicity monitoring utilizing sensitive monitoring tools is crucial for optimal audiological outcomes and mitigate the severity of ototoxicity. The monitoring protocol must be structured according to the particular patient's epidemiological characteristics. Fig. 1 shows the proposed ototoxicity monitoring protocol. Most studies have found HFA is a more sensitive tool in the early identification of ototoxic changes than PTA [24,31-33]. Length of testing is the major limitation of behavioural threshold monitoring. Due to the patient's compromised health status, it is essential to implement a quick and efficient ototoxic monitoring test protocol [34]. Studies have demonstrated the ability to detect ototoxic damage to the cochlea through a limited behavioural test frequency range, called the sensitive range of ototoxicity using PTA and HFA ( $\left.\mathrm{SRO}_{\mathrm{BEH}}\right)$. The $\mathrm{SRO}_{\mathrm{BEH}}$ is unique for each individual's audiometric configuration. The individualized $\mathrm{SRO}_{\mathrm{BEH}}$ is defined as the highest frequency with a threshold $\leq 100 \mathrm{~dB}$ 


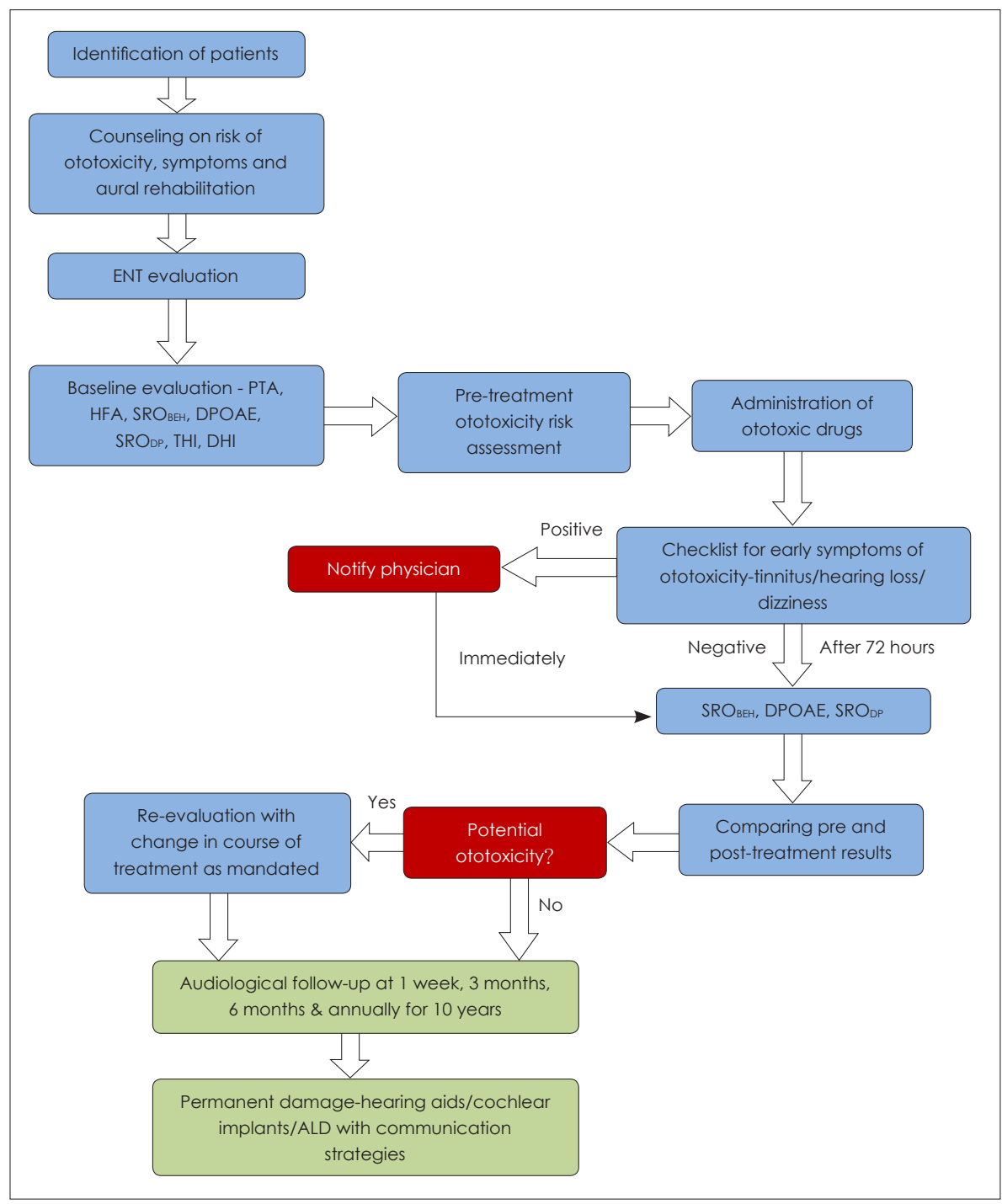

Fig. 1. Ototoxicity monitoring protocol. PTA: pure tone audiometry, HFA: high frequency audiometry, $\mathrm{SRO}_{\text {ВЕH}}$ : sensitive range of ototoxicity using PTA and HFA, DPOAE: distortion product otoacoustic emission, SRODP: sensitive range of ototoxicity using DPOAE, THI: Tinnitus Handicapped Inventory, DHI: Dizziness Handicapped Inventory, ALD: assistive listening devices.

SPL followed by 6 lower consecutive frequencies in $1 / 6$ thoctave steps.

Identifying pre-treatment patient-specific $\mathrm{SRO}_{\mathrm{BEH}}$ and then monitoring these seven frequencies, spaced at 1/6th-octave intervals for early signs of ototoxicity, reduces test duration while maintaining sensitivity, compared with PTA and HFA. Fig. 2 provides an example of a high-frequency audiogram with $\mathrm{SRO}_{\mathrm{BEH}}$ thresholds. $\mathrm{SRO}_{\mathrm{BEH}}$ is relatively quick to monitor [34-36] and can be assessed using OtoID, a portable extended high-frequency audiometer specifically designed for ototoxicity monitoring [37]. The $\mathrm{SRO}_{\mathrm{BEH}}$ is effective in identifying the frequency range in which this damage occurs [36, 38] allowing chemotherapy to be altered before significant hearing impairment occurs [38]. A substantial shift in hearing with the $\mathrm{SRO}_{\mathrm{BEH}}$ is sensitive $(90 \%)$, notwithstanding the frequency at which the shift occurs (above or below 8,000 Hz) [34-36,38]. A prospective study reported that $92 \%$ of subjects had signifi 'cant pure-tone threshold shifts within the $\mathrm{SRO}_{\mathrm{BEH}}$ region [30]. However, this behavioural monitoring procedure is more appropriate for individuals that can provide reliable behavioral responses.

ASHA and AAA guidelines for ototoxicity monitoring propose that non-behavioural measures of auditory function are necessary during baseline evaluations because patients may become incapacitated or unable to provide reliable behavioural thresholds during treatment $[23,25]$. An objective measure like DPOAEs is preferred for ototoxic monitoring compared to PTA in infants and non-responsive adults [39-41]. Few studies have examined the sensitivity of PTA, HFA, and DPOAE in the detection of ototoxicity $[30,33,42]$. A prospective study in adults investigated the ototoxic detection rate of DPOAEs and behavioural measures (HFA) and reported DPOAEs specificity rate was $78 \%$ in ears with confirmed behavioural changes and measurable DPOAEs [30]. A similar study in children 
Fig. 2. Standard high frequency audiogram, highlighting behavioural sensitivity range of ototoxicity thresholds. $\mathrm{SRO}_{\mathrm{BEH}}$ : sensitive range of ototoxicity using PTA and HFA.

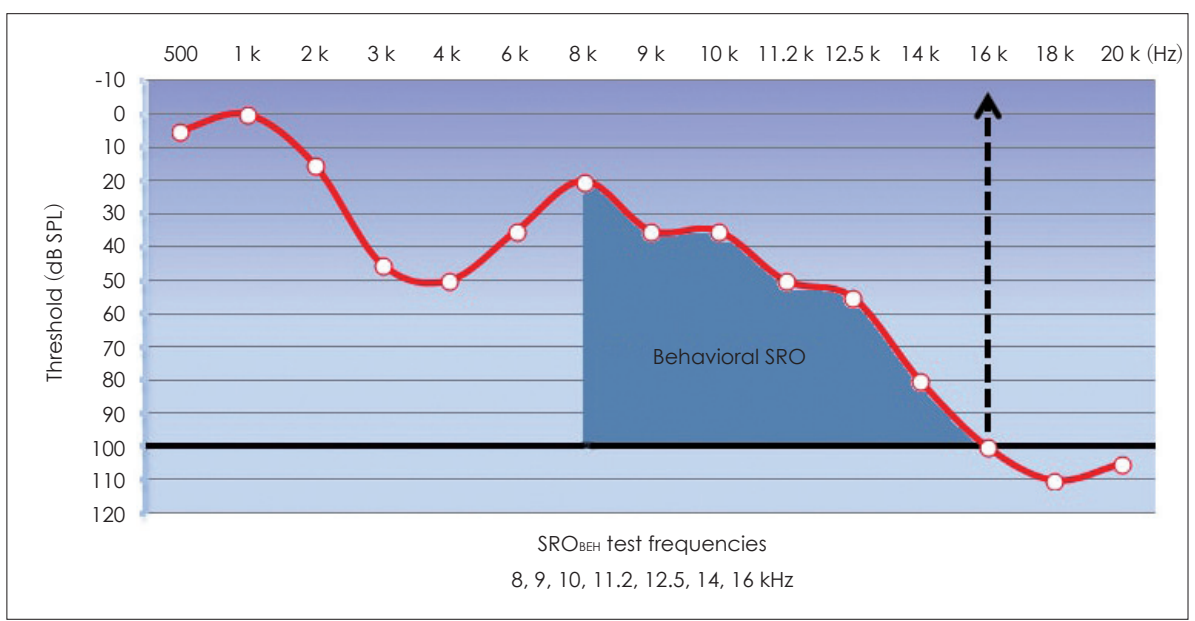

with CF who received aminoglycosides showed higher alterations in HFA compared to DPOAEs [33]. In contrast, another study concluded that both HFA and DPOAEs showed the same sensitivity in detecting ototoxicity, but did not produce the same results in all patients [42]. Knight et al. [29] concluded that ototoxic hearing shifts occurred first in HFA, then in DPOAEs, and last in the conventional PTA, while monitoring patients who received platinum-based chemotherapy.

The concept of individualized SRO has been adapted to DPOAEs, which led to a sensitive range of ototoxicity using DPOAEs (sensitive range of ototoxicity using DPOAE, $\mathrm{SRO}_{\mathrm{DP}}$ ) $[43,44]$. Results of numerous studies suggest that HFA, DPOAE, $\mathrm{SRO}_{\mathrm{BEH}}$, and $\mathrm{SRO}_{\mathrm{DP}}$ have the potential to detect incipient ototoxicity, either individually or in a combination of tests. In addition, clinical tools such as the Dizziness Handicap Inventory (DHI) and Tinnitus Handicap Inventory (THI) can be used the measure the impact of vertigo and tinnitus on a day to day life.

Advantages of non-invasive clinical tools to detect minor ototoxic changes in cochlear physiology (i.e., DPOAEs) include their frequency-specific and quantitative assessment of hearing loss and their ability to measure over broader frequency ranges. DPOAE can also be done rapidly at the bedside, with good test-retest reliability at baseline and before every ototoxic drug administration. In addition, DPOAEs are cost effective as they can reveal initial ototoxic changes before it appears on the PTA, reducing further assessment unless DPOAE results warrant further testing. One important drawback with DPOAEs is that they are sensitive to incidental middle ear dysfunction, which is common in pediatric populations and in those who are immuno-compromised by ototoxic agents. In addition, DPOAEs may not be detectable in individuals with elevated thresholds ( $>60 \mathrm{~dB}$ sound pressure level) as changes in outer hair cell amplification mechanism alter DPOAE responses and auditory thresholds. In clinical practice, measure- ment of ultra-high frequency DPOAEs requires specialized equipment to deliver and record acoustic signals. Lack of standardized calibration techniques to preclude errors at these frequencies can make it a challenging task for hearing professionals.

\section{Specific criteria for identification of ototoxicity}

Despite extensive research supporting the aforementioned audiometric tests as effective ototoxicity monitoring tools, there remains no consensus regarding the gold standard regimen for ototoxicity monitoring. Most of the ototoxicity classification systems use behavioural thresholds (PTA) as a hearing change criterion to define the occurrence of cochleotoxicity in order to assist multi-disciplinary specialists in the ototoxicity monitoring. A recent review article identified 13 key cochleotoxic classification systems using audiometric results [45]. Results indicated the common weaknesses of these grading scales are lack of sensitivity to small changes in hearing thresholds, non-inclusion of HFA, and lack of indication of which changes are likely to be clinically significant for communication and quality of life. As numerous studies have demonstrated, the sensitivity of HFA in detecting cochleotoxicity, classification systems with HFA emphasis should be utilized in practice. Among the 13 classification systems, only Chang [46] and Tune [47] included HFA into the grading system. Although the ASHA classification does not include grading, it is the only available classification emphasizing baseline evaluation and is best suited for the earliest identification of ototoxicity [23]. Table 1 shows the ototoxicity criteria with the inclusion of HFA.

The choice of effective ototoxicity monitoring method is always debatable. Even though ASHA and AAA guidelines for monitoring of ototoxicity emphasize the significance of DPOAEs, there are no universally accepted criteria to define cochleotoxicity using DPOAEs. These professional guidelines do not advocate the SRO procedure; however, these sup- 
Table 1. Ototoxicity criteria with inclusion of high frequency audiometry

\begin{tabular}{cll}
\hline Grade & \multicolumn{1}{c}{ Chang grading system } & \multicolumn{1}{c}{ Tune grading system } \\
\hline 0 & $\leq 20 \mathrm{~dB}$ at 1,2 , and $4 \mathrm{kHz}$ & No hearing loss \\
$1 \mathrm{a}$ & $\geq 40 \mathrm{~dB}$ at any frequency 6 to $12 \mathrm{kHz}$ & Threshold shift $\geq 10 \mathrm{~dB}$ at 8,10 and $12.5 \mathrm{KHz}$ \\
$1 \mathrm{~b}$ & $>20$ and $<40 \mathrm{~dB}$ at $4 \mathrm{kHz}$ & Threshold shift $\geq 10 \mathrm{~dB}$ at $1,2 \mathrm{and} 4 \mathrm{KHz}$ \\
$2 \mathrm{a}$ & $\geq 40 \mathrm{~dB}$ at $4 \mathrm{kHz}$ and above & Threshold shift $\geq 20 \mathrm{~dB}$ at $8,10 \mathrm{and} 12.5 \mathrm{KHz}$ \\
$2 \mathrm{~b}$ & $>20 \mathrm{and}<40 \mathrm{~dB}$ at any frequency below $4 \mathrm{kHz}$ & Threshold shift $\geq 20 \mathrm{~dB}$ at $1,2 \mathrm{and} 4 \mathrm{KHz}$ \\
3 & $\geq 40 \mathrm{~dB}$ at 2 or $3 \mathrm{kHz}$ and above & $\geq 35 \mathrm{~dB} \mathrm{HL}$ at $1,2 \mathrm{and} 4 \mathrm{KHz}$ \\
4 & $\geq 40 \mathrm{~dB}$ at $1 \mathrm{kHz}$ and above & $\geq 70 \mathrm{~dB} \mathrm{HL}$ at 1,2 and $4 \mathrm{KHz}$ \\
ASHA & $\geq 20 \mathrm{~dB}$ decrease in pure tone thresholds at any test frequency OR $\geq 10 \mathrm{~dB}$ decrease at two adjacent frequencies \\
& OR decreased response at three consecutive test frequencies where responses were previously obtained
\end{tabular}

ASHA emphasizes baseline evaluation for early identification of ototoxicity and Chang/Tune emphasizes documenting high frequency audiometric thresholds and grading of hearing loss. Therefore, a combination of the ASHA and Chang/Tune classifications is recommended as best practice for early identification and subsequent monitoring of ototoxicity. ASHA: American SpeechLanguage-Hearing Association, HL: hearing level.

port the inclusion of HFA in ototoxicity monitoring. Comprehensive ototoxicity monitoring program-veteran affairs have addressed these clinical problems [38]. They proposed both $\mathrm{SRO}_{\mathrm{BEH}}$ and $\mathrm{SRO}_{\mathrm{DP}}$ as the preferred ototoxicity monitoring methods, either individually or in combination, based on the patient's health condition. Significant hearing shift using ASHA's criteria within the individualized $\mathrm{SRO}_{\mathrm{BEH}}$ designates ototoxicity. A shift in DPOAE is considered significant only if the amplitude is reduced by $6 \mathrm{~dB}$ or more than the baseline $\mathrm{SRO}_{\mathrm{DP}}[38,43,44]$. DPOAEs are best utilized by assessing the measurable DPOAE $\mathrm{f} 2$ frequency range and its relation to $\mathrm{SRO}_{\text {вен. }}$

Most of the existing ototoxicity grading systems do not appreciate the effect of hearing loss at the ultra-high frequency hearing loss, which is the warning signal of incipient ototoxicity. An optimal ototoxicity classification system should be clear and unambiguous, identify and quantify the ototoxic changes with high sensitivity, reflect the relevant patient population, easy for multi-disciplinary specialities to apply and understand (audiological/otological and non-audiological/ non-otological), correlate well with functional outcomes, and have a high degree of reliability.

\section{Follow-up evaluation}

Follow up testing is recommended to identify progressive hearing changes and also to detect an improvement in hearing acuity. This can be achieved through questionnaires, documenting subjective otologic complaints, synergistic components (i.e. noise exposure, other ototoxic agents), plus subjective and objective measures of auditory function. Assessments must be scheduled at appropriate intervals allowing for early detection of ototoxicity, which can depend on cancer type and the dose and frequency of ototoxic therapy. ASHA recommends evaluations to be done in one month and 3 months following termination of ototoxic therapy [23]. At 6 months, thorough audiological evaluation is done to reassess the patient's hearing status. Clinical tools such as the Tinnitus Ototoxicity Monitoring Interview and THI can be administered to detect the onset of tinnitus or change in tinnitus characteristics while administering chemotherapy. These characteristics can be reviewed by the audiologist/medical team to make appropriate decisions. If significant hearing changes are observed for HFA, $\mathrm{SRO}_{\mathrm{BEH}}$, DPOAE, and $\mathrm{SRO}_{\mathrm{DP}}$, appropriate action needs to be taken to prevent progression and irreversible damage.

Previous studies of children and adults receiving chemotherapy have shown a progressive hearing loss with young children being more at risk than adults $[4,48,49]$. Data suggests that long-term audiologic follow-up is needed for 2 to 10 years to document recovery and plan appropriate rehabilitation [50-52].

\section{Vestibulotoxicity monitoring}

Although vestibulotoxicity of certain aminoglycosides is well established [53,54], no widely accepted guidelines for vestibulotoxicity monitoring exist. The major challenge in vestibulotoxicity monitoring is the identification of these symptoms which is apparent only when patients are mobilized and may often be incorrectly attributed to the patient's debilitated state. As there is no single test that can identify vestibulotoxicity, screening tests such as dynamic visual acuity and head impulse test, along with DHI, are recommended to monitor patients. Furthermore, vestibular diagnostic procedures are often impractical due to the patient's compromised health status. Currently, vestibular rehabilitation therapy is the preferred treatment for vestibulotoxicity but results are variable [54].

\section{Adherence to ototoxicity monitoring protocol}

In clinical practice, medical adherence is a proven key factor associated with improved short and long-term clinical 
outcomes. Factors contributing to better ototoxicity monitoring adherence are myriad and include: those that are related to the hearing healthcare system (accessibility to service, interval between diagnosis and referral, and health promotion), those that are related to professionals (effective communication among themselves, greater insight of information about adverse effects), those that are related to patients (self-efficacy, age, attitudes and beliefs, and health literacy), and those that are related to therapy-induced complications (nephrotoxicity, ototoxicity, neurotoxicity).

Medical non-adherence can adversely affect a patient's quality of life of patients while having a negative impact on their families, the cost-effectiveness of health care, clinical decisions, and the outcomes of clinical trials. While the variations in adherence to ototoxicity monitoring programs are not fatal, they certainly can negatively impact the clinical decisions and the health-related quality of life of patients. Identifying potential obstacles to ototoxicity monitoring adherence will improve adherence and health outcomes. Examples are lack of understanding of the condition, fragmented health care systems, and non-implementation of patient-centered/familycentered interventional strategies.

\section{Prophylactic Measures}

Several drugs with potential ototoxic effects are commonly used as a standard therapy in many types of cancer. These drugs often cannot be replaced easily by alternate drug regimens. Therefore, prevention of ototoxicity requires an individualized ototoxicity monitoring program in collaboration with the oncology team. This can effectively detect incipient ototoxicity early in its process so that preventive measures can be taken before the hearing loss becomes permanent and/or debilitating. Another strategy involves obtaining a personalized pre-treatment ototoxicity risk profile which would assist the healthcare professionals to predict the likely dose which produces significant hearing change as per ASHA's criteria [38]. Ototoxicity monitoring protocol should be supplemented with genetic screening to identify individuals susceptible to ototoxicity. This measure could potentially prevent hearing loss by avoiding administration of ototoxic drugs when identified potential confounders increase the risk of ototoxicity.

\section{Otoprotectants}

Many animal and in vitro studies have demonstrated the efficacy of otoprotective agents in the prevention of ototoxicity. There are a limited number of clinical trials investigating otoprotective agents. Many of them lack appropriate control groups, positive clinical findings [55-57], longitudinal out- come [58], and multicenter larger scale clinical trials [59]. Currently, otoprotective agents such as sodium thiosulfate, amifostine, and $\mathrm{N}$-acetylcysteine have been investigated for cisplatin otoprotection in clinical trials. Although systemic administration of these agents can reduce cisplatin-induced hearing loss, it also can reduce the cisplatin's antitumor efficacy [55,60-62]. Therefore, alternative approaches like delaying sodium thiosulfate for several hours after cisplatin administration plus intra-tympanic administration of these agents have been attempted to retain otoprotection and cisplatin's tumoricidal activity. A clinical study reported intra-tympanic administration of Nacetylcysteine demonstrated positive otoprotection for the prevention of cisplatin-induced ototoxicity [56]. A similar study using intra-tympanic dexamethasone also yielded positive results [63].

Although previous trials of amifostine failed to demonstrate otoprotection [61,62], a recent study reported evidence in favor of systemic administration of this drug in preventing cisplatin-induced hearing loss in medium-risk but not higherrisk medulloblastoma patients [57]. Randomized trials comparing different doses or duration of otoprotective agents are lacking. A Cochrane review of 3 randomized, controlled trials of amifostine agents reported that no conclusions can be made about their efficacy in preventing cisplatin-induced ototoxicity in children [64]. Currently, no drugs have been given approval by the US Food and Drug Administration for prevention of drug-induced ototoxicity during curative cancer treatment [65]. Additional clinical research is needed to study the otoprotective nature of these drugs.

In recent decades, successful promotion of cochlear gene therapy, adeno-associated virus-mediated delivery of brainderived neurotrophic factor, and stem cells have been thoroughly demonstrated in animal models $[66,67]$. With further improvement, these approaches may become clinically applicable over the coming decade.

\section{Rehabilitation of Ototoxicity}

The damage and/or disability of drug-induced hearing loss are often permanent and, therefore, it is important to minimize further damage. In case of permanent ototoxic damage and pre-exposure hearing loss, aural rehabilitation should be considered such as amplification devices/cochlear implants/ assistive listening devices in conjunction with communication strategies. Often, similar audiological characteristics or even patients with normal audiograms exhibit varying degrees of communication difficulties. Therefore, rehabilitation must be based on the communication difficulties rather than audiological outcomes. Self-reported measures of communication 
difficulty are recommended to guide treatment plans and gain a greater understanding of the incidence and burden of ototoxicity.

\section{Areas of Uncertainty}

Although many ototoxic drugs are reported in the literature, a standardized classification of these drugs is unavailable. Therefore, it is difficult for medical professionals to categorize which patients require pre-treatment audiological screening for a certain class of drugs. A pre-treatment screening may not be feasible in patients with poor physical health. Furthermore, genetic susceptibility plays a major role in ototoxicity. The initial effects of ototoxicity may go unnoticed by patients, caretakers, and medical professionals. There is also an absence of established protocols in vestibulotoxicity monitoring for patients who are critically ill. Furthermore, the equipment to measure HFA is not readily available in many clinics. All of these factors make early diagnosis and treatment of ototoxicity very difficult to achieve.

\section{Conclusion}

Ototoxicity is considered an otologic urgency because there is less recovery of functional damage when a treatment plan is not implemented promptly. Once the ototoxic medication is administered, regular monitoring should be a proactive step. A comprehensive assessment of ototoxicity should include sensitive audiological tests such as audiometry and DPOAEs that assess ultra-high frequencies and appropriate ototoxic grading criteria with high sensitivity and specificity. In clinical practice, individuals with a pre-exposure hearing loss should require the judicial use of ototoxic drugs and alternative treatment regimens should be employed. A robust ototoxicity monitoring protocol requires synergistic relationships between oncologists, audiologists, and otologists including positive patientclinician relationships. This team approach would foster truly patient/family-centered rehabilitation. Improved understanding of the cellular and molecular underpinnings of ototoxicity is critical to developing individualized preventive and rehabilitative strategies, thus minimizing chronic morbidities and optimizing the health-related quality of life. There is a greater need for clinical trials which can translate its outcomes into everyday practice aiming to enhance the adoption of best practices in the community and for international guidelines for ototoxicity monitoring.

\section{Conflicts of interest}

The authors have no financial conflicts of interest.

\section{REFERENCES}

1) Naunton RF, Ward PH. The ototoxicity of Kanamycin sulfate in the presence of compromised renal function. AMA Arch Otolaryngol 1959;69:398-9.

2) Arslan E, Orzan E, Santarelli R. Global problem of drug-induced hearing loss. Ann N Y Acad Sci 1999;884:1-14.

3) Cianfrone G, Pentangelo D, Cianfrone F, Mazzei F, Turchetta R, Orlando MP, et al. Pharmacological drugs inducing ototoxicity, vestibular symptoms and tinnitus: a reasoned and updated guide. Eur Rev Med Pharmacol Sci 2011;15:601-36.

4) Knight KR, Kraemer DF, Neuwelt EA. Ototoxicity in children receiving platinum chemotherapy: underestimating a commonly occurring toxicity that may influence academic and social development. J Clin Oncol 2005;23:8588-96.

5) Konrad-Martin D, Gordon JS, Reavis K, Wilmington DJ, Helt WJ, Fausti SA. Audiological monitoring of patients receiving ototoxic drugs. Perspect Hear Hear Disord Res Res Diagn 2005;9:17-22.

6) Coradini PP, Cigana L, Selistre SG, Rosito LS, Brunetto AL. Ototoxicity from cisplatin therapy in childhood cancer. J Pediatr Hematol Oncol 2007;29:355-60.

7) McKeage MJ. Comparative adverse effect profiles of platinum drugs. Drug Saf 1995;13:228-44.

8) Bisht M, Bist SS. Ototoxicity: the hidden menace. Indian J Otolaryngol Head Neck Surg 2011;63:255-9.

9) Rybak LP. Ototoxicity of loop diuretics. Otolaryngol Clin North Am 1993;26:829-44.

10) Peters U, Preisler-Adams S, Hebeisen A, Hahn M, Seifert E, Lanvers $\mathrm{C}$, et al. Glutathione S-transferase genetic polymorphisms and individual sensitivity to the ototoxic effect of cisplatin. Anticancer Drugs 2000;11:639-43.

11) Oldenburg J, Kraggerud SM, Cvancarova M, Lothe RA, Fossa SD. Cisplatin-induced long-term hearing impairment is associated with specific glutathione s-transferase genotypes in testicular cancer survivors. J Clin Oncol 2007;25:708-14.

12) Riedemann L, Lanvers C, Deuster D, Peters U, Boos J, Jürgens H, et al. Megalin genetic polymorphisms and individual sensitivity to the ototoxic effect of cisplatin. Pharmacogenomics J 2008;8:23-8.

13) Ross CJ, Katzov-Eckert H, Dubé MP, Brooks B, Rassekh SR, Barhdadi A, et al.; CPNDS Consortium. Genetic variants in TPMT and COMT are associated with hearing loss in children receiving cisplatin chemotherapy. Nat Genet 2009;41:1345-9.

14) Palodetto B, Postal M, Grignoli CR, Sartorato EL, Oliveira CA. Influence of glutathione s-transferase on the ototoxicity caused by aminoglycosides. Braz J Otorhinolaryngol 2010;76:306-9.

15) Rednam S, Scheurer ME, Adesina A, Lau CC, Okcu MF. Glutathione S-transferase P1 single nucleotide polymorphism predicts permanent ototoxicity in children with medulloblastoma. Pediatr Blood Cancer 2013;60:593-8.

16) Choeyprasert W, Sawangpanich R, Lertsukprasert K, Udomsubpayakul U, Songdej D, Unurathapan U, et al. Cisplatin-induced ototoxicity in pediatric solid tumors: the role of glutathione S-transferases and megalin genetic polymorphisms. J Pediatr Hematol Oncol 2013; 35:e138-43.

17) Pussegoda K, Ross CJ, Visscher H, Yazdanpanah M, Brooks B, Rassekh SR, et al. Replication of TPMT and $\mathrm{ABCC} 3$ genetic variants highly associated with cisplatin-induced hearing loss in children. Clin Pharmacol Ther 2013;94:243-51.

18) Yang JJ, Lim JY, Huang J, Bass J, Wu J, Wang C, et al. The role of inherited TPMT and COMT genetic variation in cisplatin-induced ototoxicity in children with cancer. Clin Pharmacol Ther 2013;94: 252-9.

19) Hagleitner MM, Coenen MJ, Patino-Garcia A, de Bont ES, Gonzalez-Neira A, Vos HI, et al. Influence of genetic variants in TPMT and COMT associated with cisplatin induced hearing loss in patients with cancer: two new cohorts and a meta-analysis reveal significant 
heterogeneity between cohorts. PLoS One 2014;9:e115869.

20) Qian Y, Guan MX. Interaction of aminoglycosides with human mitochondrial $12 \mathrm{~S}$ rRNA carrying the deafness-associated mutation. Antimicrob Agents Chemother 2009;53:4612-8.

21) Xu H, Robinson GW, Huang J, Lim JY, Zhang H, Bass JK, et al. Common variants in ACYP2 influence susceptibility to cisplatininduced hearing loss. Nat Genet 2015;47:263-6.

22) Brown AL, Lupo PJ, Okcu MF, Lau CC, Rednam S, Scheurer ME. SOD2 genetic variant associated with treatment-related ototoxicity in cisplatin-treated pediatric medulloblastoma. Cancer Med 2015; 4:1679-86.

23) American Speech-Language-Hearing Association. Guidelines for the audiologic management of individuals receiving cochleotoxic drug therapy. ASHA 1994;36(Suppl 12):11-9.

24) Fausti SA, Larson VD, Noffsinger D, Wilson RH, Phillips DS, Fowler CG. High-frequency audiometric monitoring strategies for early detection of ototoxicity. Ear Hear 1994;15:232-9.

25) American Academy of Audiology position statement and clinical practice guidelines: ototoxicity monitoring 2009 [cited 2016 Jan 5]. Available from: http://www.audiology.org/publications-resources/ document-library/ototoxicity-monitoring.

26) Wilkinson JD, Mora J. Audit of hearing surveillance in UK paediatric cystic fibrosis units. J Cyst Fibros 2009;8 Suppl 2:S107.

27) Van Meter DJ, Corriveau M, Ahern JW, Lahiri T. A survey of oncedaily dosage tobramycin therapy in patients with cystic fibrosis. Pediatr Pulmonol 2009;44:325-9.

28) Al-Malky G. An analysis of ototoxicity in children: audiological detection, clinical practice and genetic susceptibility [dissertation]. London: University College London (UCL);2014.

29) Knight KR, Kraemer DF, Winter C, Neuwelt EA. Early changes in auditory function as a result of platinum chemotherapy: use of extended high-frequency audiometry and evoked distortion product otoacoustic emissions. J Clin Oncol 2007;25:1190-5.

30) Reavis KM, Phillips DS, Fausti SA, Gordon JS, Helt WJ, Wilmington $\mathrm{D}$, et al. Factors affecting sensitivity of distortion-product otoacoustic emissions to ototoxic hearing loss. Ear Hear 2008;29:875-93.

31) Fausti SA, Henry JA, Schaffer HI, Olson DJ, Frey RH, Bagby GC Jr. High-frequency monitoring for early detection of cisplatin ototoxicity. Arch Otolaryngol Head Neck Surg 1993;119:661-6.

32) Abujamra AL, Escosteguy JR, Dall'Igna C, Manica D, Cigana LF, Coradini $\mathrm{P}$, et al. The use of high-frequency audiometry increases the diagnosis of asymptomatic hearing loss in pediatric patients treated with cisplatin-based chemotherapy. Pediatr Blood Cancer 2013;60: 474-8.

33) Geyer LB, Menna Barreto SS, Weigert LL, Teixeira AR. High frequency hearing thresholds and product distortion otoacoustic emissions in cystic fibrosis patients. Braz J Otorhinolaryngol 2015;81: 589-97.

34) Fausti SA, Helt WJ, Phillips DS, Gordon JS, Bratt GW, Sugiura KM, et al. Early detection of ototoxicity using 1/6th-octave steps. J Am Acad Audiol 2003;14:444-50.

35) Fausti SA, Henry JA, Helt WJ, Phillips DS, Frey RH, Noffsinger D, et al. An individualized, sensitive frequency range for early detection of ototoxicity. Ear Hear 1999;20:497-505.

36) Vaughan NE, Fausti SA, Chelius S, Phillips D, Helt W, Henry JA. An efficient test protocol for identification of a limited, sensitive frequency test range for early detection of ototoxicity. J Rehabil Res Dev 2002;39:567-74.

37) Dille MF, Jacobs PG, Gordon SY, Helt WJ, McMillan GP. OtoID: new extended frequency, portable audiometer for ototoxicity monitoring. J Rehabil Res Dev 2013;50:997-1006.

38) Konrad-Martin D, Reavis KM, McMillan G, Helt WJ, Dille M. Proposed comprehensive ototoxicity monitoring program for VA healthcare (COMP-VA). J Rehabil Res Dev 2014;51:81-100.

39) Stavroulaki P, Vossinakis IC, Dinopoulou D, Doudounakis S, Adamopoulos G, Apostolopoulos N. Otoacoustic emissions for moni- toring aminoglycoside-induced ototoxicity in children with cystic fibrosis. Arch Otolaryngol Head Neck Surg 2002;128:150-5.

40) Yilmaz S, Oktem F, Karaman E. Detection of cisplatin-induced ototoxicity with transient evoked otoacoustic emission test before pure tone audiometer. Eur Arch Otorhinolaryngol 2010;267:1041-4.

41) Md Daud MK, Mohamadl H, Haron A, Rahman NA. Ototoxicity screening of patients treated with streptomycin using distortion product otoacoustic emissions. B-ENT 2014;10:53-8.

42) Yu KK, Choi CH, An YH, Kwak MY, Gong SJ, Yoon SW, et al. Comparison of the effectiveness of monitoring cisplatin-induced ototoxicity with extended high-frequency pure-tone audiometry or distortion-product otoacoustic emission. Korean J Audiol 2014;18:58-68.

43) Dille MF, McMillan GP, Reavis KM, Jacobs P, Fausti SA, KonradMartin D. Ototoxicity risk assessment combining distortion product otoacoustic emissions with a cisplatin dose model. J Acoust Soc Am 2010;128:1163-74

44) Reavis KM, McMillan G, Austin D, Gallun F, Fausti SA, Gordon JS, et al. Distortion-product otoacoustic emission test performance for ototoxicity monitoring. Ear Hear 2011;32:61-74.

45) Crundwell G, Gomersall P, Baguley DM. Ototoxicity (cochleotoxicity) classifications: a review. Int J Audiol 2016;55:65-74.

46) Chang KW, Chinosornvatana N. Practical grading system for evaluating cisplatin ototoxicity in children. J Clin Oncol 2010;28:1788-95.

47) Theunissen EA, Dreschler WA, Latenstein MN, Rasch CR, van der Baan S, de Boer JP, et al. A new grading system for ototoxicity in adults. Ann Otol Rhinol Laryngol 2014;123:711-8.

48) Kushner BH, Budnick A, Kramer K, Modak S, Cheung NK. Ototoxicity from high-dose use of platinum compounds in patients with neuroblastoma. Cancer 2006;107:417-22.

49) Yancey A, Harris MS, Egbelakin A, Gilbert J, Pisoni DB, Renbarger J. Risk factors for cisplatin-associated ototoxicity in pediatric oncology patients. Pediatr Blood Cancer 2012;59:144-8

50) Bertolini P, Lassalle M, Mercier G, Raquin MA, Izzi G, Corradini N, et al. Platinum compound-related ototoxicity in children: long-term follow-up reveals continuous worsening of hearing loss. J Pediatr Hematol Oncol 2004;26:649-55.

51) Al-Khatib T, Cohen N, Carret AS, Daniel S. Cisplatinum ototoxicity in children, long-term follow up. Int J Pediatr Otorhinolaryngol 2010; 74:913-9.

52) Kolinsky DC, Hayashi SS, Karzon R, Mao J, Hayashi RJ. Late onset hearing loss: a significant complication of cancer survivors treated with cisplatin containing chemotherapy regimens. J Pediatr Hematol Oncol 2010;32:119-23.

53) Ahmed RM, Hannigan IP, MacDougall HG, Chan RC, Halmagyi GM. Gentamicin ototoxicity: a 23-year selected case series of 103 patients. Med J Aust 2012;196:701-4.

54) Rogers C, Petersen L. Aminoglycoside-induced balance deficits: a review of vestibulotoxicity. S Afr Fam Pract 2011;53:419-24.

55) Fouladi M, Chintagumpala M, Ashley D, Kellie S, Gururangan S, Hassall T, et al. Amifostine protects against cisplatin-induced ototoxicity in children with average-risk medulloblastoma. J Clin Oncol 2008;26:3749-55.

56) Riga MG, Chelis L, Kakolyris S, Papadopoulos S, Stathakidou S, Chamalidou E, et al. Transtympanic injections of $\mathrm{N}$-acetylcysteine for the prevention of cisplatin-induced ototoxicity: a feasible method with promising efficacy. Am J Clin Oncol 2013;36:1-6.

57) Gurney JG, Bass JK, Onar-Thomas A, Huang J, Chintagumpala M, Bouffet E, et al. Evaluation of amifostine for protection against cisplatin-induced serious hearing loss in children treated for average-risk or high-risk medulloblastoma. Neuro Oncol 2014;16:848-55.

58) Dias MA, Sampaio AL, Venosa AR, Meneses Ede A, Oliveira CA. The chemopreventive effect of Ginkgo biloba extract 761 against cisplatin ototoxicity: a pilot study. Int Tinnitus J 2015;19:12-9.

59) Feldman L, Efrati S, Eviatar E, Abramsohn R, Yarovoy I, Gersch E, et al. Gentamicin-induced ototoxicity in hemodialysis patients is ameliorated by N-acetylcysteine. Kidney Int 2007;72:359-63. 
60) Doolittle ND, Muldoon LL, Brummett RE, Tyson RM, Lacy C, Bubalo JS, et al. Delayed sodium thiosulfate as an otoprotectant against carboplatin-induced hearing loss in patients with malignant brain tumors. Clin Cancer Res 2001;7:493-500.

61) Marina N, Chang KW, Malogolowkin M, London WB, Frazier AL, Womer RB, et al.; Children's Oncology Group. Amifostine does not protect against the ototoxicity of high-dose cisplatin combined with etoposide and bleomycin in pediatric germ-cell tumors: a Children's Oncology Group study. Cancer 2005;104:841-7.

62) Katzenstein HM, Chang KW, Krailo M, Chen Z, Finegold MJ, Rowland J, et al. Amifostine does not prevent platinum-induced hearing loss associated with the treatment of children with hepatoblastoma: a report of the Intergroup Hepatoblastoma Study P9645 as a part of the Children's Oncology Group. Cancer 2009;115:5828-35.
63) Marshak T, Steiner M, Kaminer M, Levy L, Shupak A. Prevention of cisplatin-induced hearing loss by intratympanic dexamethasone: a Randomized Controlled Study. Otolaryngol Head Neck Surg 2014; 150:983-90.

64) van As JW, van den Berg H, van Dalen EC. Medical interventions for the prevention of platinum-induced hearing loss in children with cancer. Cochrane Database Syst Rev 2014:CD009219.

65) Landier W. Ototoxicity and cancer therapy. Cancer 2016;122:164758.

66) Lustig LR, Akil O. Cochlear gene therapy. Curr Opin Neurol 2012; 25:57-60.

67) Santaolalla F, Salvador C, Martínez A, Sánchez JM, Del Rey AS. Inner ear hair cell regeneration: a look from the past to the future. Neural Regen Res 2013;8:2284-9. 